\title{
A STUDY OF RICOCHET PHENOMENON FOR INCLINED IMPACT OF PROJECTILE
}

\author{
JONG-HYUN JO \\ Dept. of Mechanical Design Engineering, Chungnam National University \\ 220 Gung-dong, Yuseong-gu, Daejeon, 305-764, South Korea \\ talljojo@naver.com \\ YOUNG-SHIN LEE \\ Dept. of Mechanical Design Engineering, Chungnam National University \\ 220 Gung-dong, Yuseong-gu, Daejeon, 305-764, South Korea \\ Corresponding author,leeys@cnu.ac.kr
}

\begin{abstract}
In this study, the numerical simulation using AUTODYN-3D program was investigated for trajectory prediction for inclined impacts of projectiles. The penetration and perforation of polycarbonate(PC) plate by $7.62 \mathrm{~mm}$ projectile was investigated numerically. The characteristic structure of the projectile's trajectory in the PC plates was studied. Two combined failure criteria were used in the target plate, and the target plate was modeled with the properties of polycarbonate for simulating the ricochet phenomenon. The numerical analyses were used to study the effect of the angle of inclination on the trajectory and kinetic energy of the projectile. The dynamic deformation behaviors tests of PC were compared with numerical simulation results which can be used for predictive purpose. Ricochet phenomenon for angles of inclination of $0^{\circ} \leq$ $\theta \leq 20^{\circ}$ in the analysis. The projectile perforated the plate for $\theta>30^{\circ}$, thus defined a failure envelope for numerical configuration. The numerical analyses was used to study the effect under the projectile impact velocity on the depth of penetration(DOP).
\end{abstract}

Keywords: Projectile, trajectory prediction, ricochet phenomenon.

\section{Introduction}

The penetration including the perforation and ricochet phenomenon occurs under high velocity impact. The phenomenon of ricochet is an important consideration in the impacting projectile. The impact and perforation of polymethylmethacrylate(PMMA) plates have been the subject of recent investigations by Rosenberg et al. [1] who showed an interesting ricochet phenomenon that occurs for inclined impacts. These authors presented an extensive experimental and numerical study, with the main conclusion that spalling (dynamic tensile failure) is indeed the governing factor in the generation of the ricochet[1-2]. Recently, the mechanical properties of polymers at high strain rates and confinement were investigated: polycarbonate (PC) by Rittel and Dorogoy [3]. Polycarbonate (PC) is well known for its high ballistic resistance to penetration and 
perforation, and is widely used in impact-resistant applications. Hence numerous investigations have been carried out to characterize its static and dynamic flow and failure properties (e.g. Ravi-Chandar [4], Rittel et al. [5-6] and Sarva et al. [7]).

High velocity impacting test were done by Sung-Taek Park et al.[8] using PC plate. Then the dynamic deformation behaviors of PC were studied under various impact velocities. As a result, the PC showed typical mushroom head as shown in high impact behaviors of metal materials and the attack face showed ductile behavior. The ductile fracture performance of polycarbonate was investigated by Jeffrey A [9]. Hence, this investigation addresses the inclined ballistic impact of polycarbonate plates by a $7.62 \mathrm{~mm}$ projectile under the combined effects of tensile and ductile fracture performance(fracture energy).

The dynamic deformation behaviors tests of PC were compared with numerical simulation results which can be used as predictive purpose.

\section{Numerical simulation}

The idea is to develop an ultimately predictive model to help in design, minimize the number of ballistic tests, also provide insight into the penetration process. The type of analysis, geometry, material properties, failure criteria are detailed in the sequel. A prediction for the depth of penetration is also given.

\subsection{Analysis}

The numerical simulations were carried out using AUTODYN-3D explicit finite element code [10]. The hydrodynamic continuum equations are derived from application of the conservation laws of mass, momentum, and energy. An equation of state provides the relationship between pressure, density, and internal energy. An elastic-plastic Mises plasticity model is used for constitutive relations. The constitutive behavior of polycarbonate was represented using a hydrodynamic material model in which the material's volumetric strength is determined by a linear Miee Grüneisen equations of state [10], while the deviatoric behavior is of the isotropic elastic-plastic Mises-type. Two failure criteria [10]: (1) tensile failure (2) ductile fracture performance, were used.

\subsection{Material properties}

The copper jacket and steel core was modeled as an elastic-plastic material with ductile fracture performance [10]. Finally, a hydrodynamic material model in which the material's volumetric strength is determined by an equation of state was used for the polycarbonate plate, namely the Shock equation of state based on Mie-Gruneisen with application of linear $U_{S}-U_{P}$ Hugoniot. $U_{S}$ is the linear shock wave and $U_{P}$ is the particle speed.

This model can be applied to materials which also have isotropic elastic or viscous deviatoric behavior. It is used with a Mises plasticity model. More details can be found in Autodyn Analysis User's Manual in chapter: equation of state [10]. 
Table 1. Physical properties of the materials modeled in the simulation.

\begin{tabular}{lllll}
\hline Material & $\rho\left[\mathrm{g} / \mathrm{cm}^{3}\right]$ & Shear modulus[GPa] & Yield stess[MPa] & Poisson's ratio \\
\hline Steel V250 & 8.12 & 71.80 & 1560 & 0.29 \\
Cart Brass & 8.45 & 37.40 & 112.0 & 0.375 \\
Polycarbonate & 1.20 & 1 & 80.60 & 0.36 \\
\hline
\end{tabular}

Table 2. Hydrodynamic data for materials.

\begin{tabular}{lll}
\hline Material & Grueisen coefficient & Specific heat $\left[\mathrm{J} / \mathrm{kg} \mathrm{K}^{\circ}\right]$ \\
\hline Steel V250 & 1.6 & 408 \\
Cart Brass & 2.04 & 385 \\
Polycarbonate & 0.61 & 1300 \\
\hline
\end{tabular}

The hydrodynamic material data for the polycarbonate was taken from [10] who used the $A U T O D Y N^{T M}$ [10] material libraries. The physical properties of the materials are summarized in Table 1. The hydrodynamic data for the polycarbonate are given in Table 2.

\subsection{Failure criteria}

Two failure criteria which are available in AUTODYN-3D [10] were used for 1) tensile failure and 2) ductile fracture performance.

\subsection{Data for failure criteria}

The tensile failure value was set to $187 \mathrm{MPa}$. The linear displacement fracture energy value was set to $80 \mu \mathrm{m}$. A constant value of plastic strain of 0.4 was used for all triaxialities and strain rates with a zero linear displacement fracture energy.

\section{Numerical results}

\subsection{Experimental verification of numerical simulation}

Sung-Taek Park et al.[8] did the Taylor Impact tests with cylindrical polymeric materials such as PE, PC and PEEK. They studied deformation and fracture behaviors of polymeric materials when high speed polymeric strike the target. Also, the deformation behaviors were compared and discussed under various impact velocities. The specimen used in the test with a diameter of $10 \mathrm{~mm}$, long of $50 \mathrm{~mm}$ and density of $1.2 \mathrm{~g} / \mathrm{cm}^{3}$. The tests were done for studying the deformation behaviors under various impact velocities. Dynamic Simulations for deformation behavior of PC were done with various high velocity impacts to confirm the numerical simulation used in this study under the same conditions of test. Fig. 1 shows the deformation length $L$ and plastic deformation $\lambda$ after the impact test [8]. 


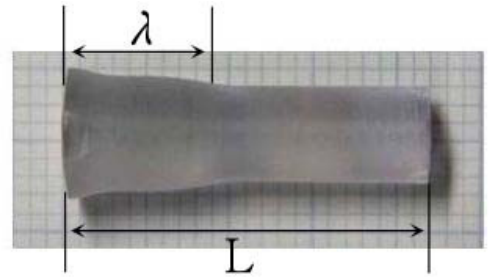

Fig. 1. Change rate in cylinder length. $\mathrm{PC}$ at $\mathrm{V}=232 \mathrm{~m} / \mathrm{s}$

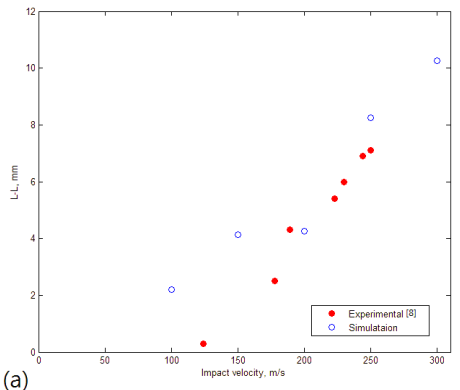



Fig. 2. Comparison of simulation with the recovered cylinder for a test on $50 \mathrm{~mm}$ cylinder at $200 \mathrm{~m} / \mathrm{s}$.

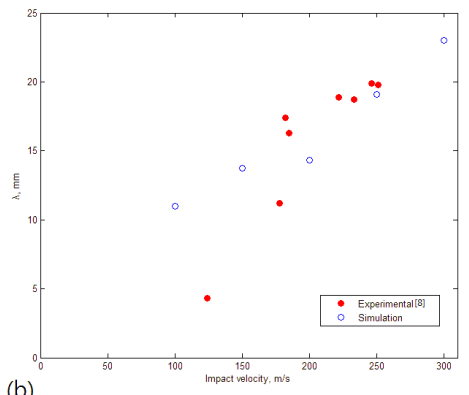

(b)

Fig. 3. Change rate in cylinder length, (a) $\mathrm{L}_{0}-\mathrm{L}$ rate (b) Size of $\lambda$

Fig. 2 shows the comparisons of test and numerical simulation deflection of polymeric materials PC at $200 \mathrm{~m} / \mathrm{s}$. As Fig. 3 shows, the deformation of test and numerical simulation got the similar pattern when the impact velocity above $200 \mathrm{~m} / \mathrm{s}$. In test and numerical simulation both cases, the higher the impact velocity the higher spring-back effect after mushroom head form. Also the stepwise deposition of projectile can be seen. The results displayed as the attack face showed ductile behavior.

The results show that the test values agree satisfactorily with the experimental values, so that the angle of inclination effects on the ricochet phenomenon were studied numerically.

\subsection{Effect of angle of inclination on the projectile trajectory}

The numerically obtained trajectories for angles of inclinations: $0^{\circ} \leq \theta \leq 90^{\circ}$ can be divided into three regions: 1) $0^{\circ} \leq \theta \leq 20^{\circ}$ ricochet, 2) $20^{\circ}<\theta \leq 30^{\circ}$ penetration and arrest, 3) $\theta>30^{\circ}$ full perforation.

The numerically obtained trajectories at $\theta=10^{\circ}, 12^{\circ}, 14^{\circ}, 16^{\circ}, 18^{\circ}, 20^{\circ}$ and $\theta=22^{\circ}, 24^{\circ}$, $26^{\circ}, 28^{\circ}, 30^{\circ}, 50^{\circ}, 70^{\circ}$ inclination were shown in Fig. 4a-f and Fig. 5a-g respectively.

Numerically, the highest angle of inclination was calculated as $20^{\circ}$ to cause the ricochet. For $20^{\circ}<\theta \leq 30^{\circ}$ the projectile can penetrate the plate but can not perforate it. For angles $\theta=50^{\circ}$ and $\theta=70^{\circ}$, the trajectory were straight lines since the initial vertical components of velocities were high. Also, the projectile can perforate the plate because the tensile failure effect can not supply enough influential to alter its direction. 


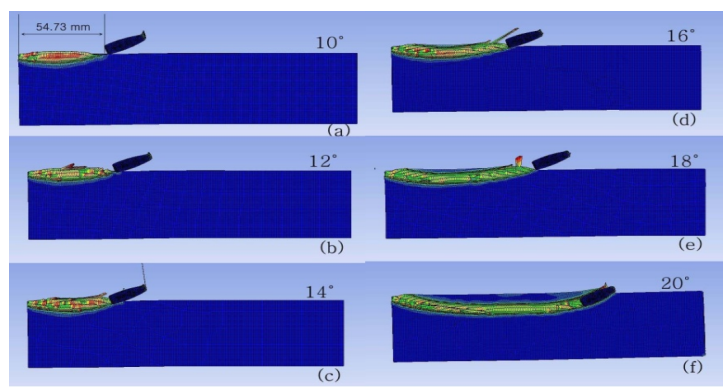

Fig. 4. Trajectories of a projectile impacting a polycarbonate plate at different angles of inclination: $10^{\circ} \sim 20^{\circ}$



Fig. 5. Trajectories of a projectile impacting a polycarbonate plate at different angles of inclination: $22^{\circ} \sim 70^{\circ}$.

\subsection{The maximum depth of penetration (DOP)}

Five numerical analyses at five different impact velocities: 200, 400, 600, 800 and 1000 $\mathrm{m} / \mathrm{s}$ were performed. The PC plate was $200 \mathrm{~mm}$ long, $20 \mathrm{~mm}$ thick and $50 \mathrm{~mm}$ wide. Because of symmetry, only one half of the physical domain was modeled. The results of the corresponding DOP were plotted in Fig. 6.

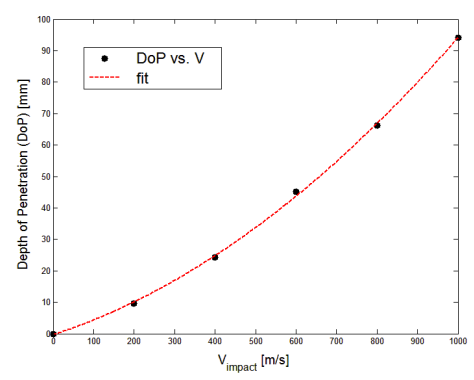

Fig. 6. The DOP vs impact velocity for normal penetration in PC.

It can be noted that the DOP scales almost linearly with the impact velocity higher than $200 \mathrm{~m} / \mathrm{s}$. Impact velocity versus residual velocity were calculated and Fig. 7 shows penetration velocity versus the residual velocity. 


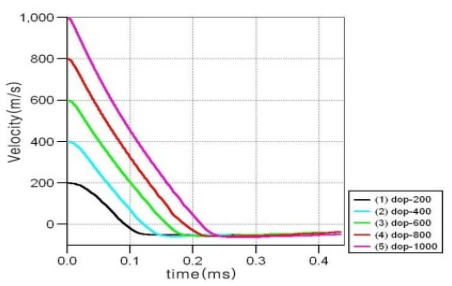

Fig. 7. Comparison of residual velocity of projectile with various impact velocities.

It can be observed that the residual velocities were almost linear relative to penetration velocities. It's mean that they were received high impact resistance under higher velocity conditions.

\section{Conclusion}

The conclusions can be summarized as follows:

(1) The combined effect of the two failure criteria (tensile and ductile fracture performance) can well predict the projectile trajectory within a PC plate.

(2) The main contributor to the trajectory within the PC was the "ductile fracture performance" criterion. Additional effect of "tensile failure" influenced the direction and depth of the projectile.

(3) The loss of kinetic energy was decreased as linear function of time during inclined penetration.

(4) The residual velocity was almost linear relative to penetration velocity and got higher value with higher velocity during penetration for all impact velocities.

\section{References}

1. Rosenberg Z, Surujon Z, Yeshurun Y, Ashuach Y and Dekel E, "Ricochet of 0.3" AP projectile from inclined polymeric plates", Int. J. Impact Eng, Vol.31, No.3, pp.221-233, 2005.

2. Dorogoy A, Rittel D and Brill A, "A study of inclined impact in polymethylmethacrylate plates", Int. J. Impact Eng, Vol.37, No.3, pp.285-294, 2010.

3. Rittel D and Dorogoy A, "A methodology to assess the rate and pressure sensitivity of polymers over a wide range of strain rates", J. Mech Phys Solids, Vol.56, No.11, pp.31913205, 2008.

4. Ravi-Chandar K, "On the failure mode transitions in polycarbonate under dynamic mixedmode loading", Int. J. Solids Struct, Vol.32, No.6-7, pp.925-938, 1995.

5. Rittel D, Levin R and Maigre H, "On dynamic crack initiation in polycarbonate under mixedmode loading", Mech Res Commun, Vol.24, No.1, pp.57-64, 1997.

6. Rittel D and Levin R, "Mode-mixity and dynamic failure mode transitions in polycarbonate", Mech Mater, Vol.30, No.3, pp.197-216, 1998.

7. Sarva S and Mulliken AD, "Mechanic of Taylor impact testing of polycarbonate", Int J Solids Struct, Vol.44, No.7-8, pp.2381-400, 2007.

8. Sung-Taek Park, Jung-Soo Park, Hyung-Seop Shin, Joon-Hong Choi and Jeong-Tae Kim, "Analysis of dynamic behavior in polymeric materials using high-speed photography during Taylor impact", Proceeding of KSME 97 Fall Conference, Vol.5, pp.2607-2612, 2007.

9. Jeffrey A, "Fractographic characterization of polycarbonate failure modes", Stork Technimet, Inc, 2004.

10. ANSYS/Autodyn 12.1 user's manual, (chapters Material models). 